\title{
Cost-effectiveness of stereotactic radiosurgery with and without whole-brain radiotherapy for the treatment of newly diagnosed brain metastases
}

\author{
Clinical article
}

\author{
MatThew D. Hall, M.D., M.B.A., ${ }^{1,4}$ James L. McGeE, M.D., S.M., ${ }^{1,3}$ \\ Mackenzie C. McGee, M.D., ${ }^{1,3}$ Kevin A. Hall, B.S., B.A., ${ }^{1}$ David M. Neils, M.D., ${ }^{2}$ \\ Jeffrey D. Klopfenstein, M.D., ${ }^{2,3}$ And Patrick W. Elwood, M.D. ${ }^{2,3}$ \\ Departments of ${ }^{1}$ Radiology and ${ }^{2}$ Neurosurgery, University of Illinois College of Medicine at Peoria, Peoria, \\ Illinois; ${ }^{3}$ Illinois Neurological Institute, Peoria, Illinois; and ${ }^{4}$ Department of Radiation Oncology, City of \\ Hope National Medical Center, Duarte, California
}

Object. Stereotactic radiosurgery (SRS) alone is increasingly used in patients with newly diagnosed brain metastases. Stereotactic radiosurgery used together with whole-brain radiotherapy (WBRT) reduces intracranial failure rates, but this combination also causes greater neurocognitive toxicity and does not improve survival. Critics of SRS alone contend that deferring WBRT results in an increased need for salvage therapy and in higher costs. The authors compared the cost-effectiveness of treatment with SRS alone, SRS and WBRT (SRS+WBRT), and surgery followed by SRS (S+SRS) at the authors' institution.

Methods. The authors retrospectively reviewed the medical records of 289 patients in whom brain metastases were newly diagnosed and who were treated between May 2001 and December 2007. Overall survival curves were plotted using the Kaplan-Meier method. Multivariate proportional hazards analysis (MVA) was used to identify factors associated with overall survival. Survival data were complete for $96.2 \%$ of patients, and comprehensive data on the resource use for imaging, hospitalizations, and salvage therapies were available from the medical records. Treatment costs included the cost of initial and all salvage therapies for brain metastases, hospitalizations, management of complications, and imaging. They were computed on the basis of the 2007 Medicare fee schedule from a payer perspective. Average treatment cost and average cost per month of median survival were compared. Sensitivity analysis was performed to examine the impact of variations in key cost variables.

Results. No significant differences in overall survival were observed among patients treated with SRS alone, SRS+WBRT, or S+SRS with respective median survival of 9.8, 7.4, and 10.6 months. The MVA detected a significant association of overall survival with female sex, Karnofsky Performance Scale (KPS) score, primary tumor control, absence of extracranial metastases, and number of brain metastases. Salvage therapy was required in $43 \%$ of SRSalone and $26 \%$ of SRS+WBRT patients $(\mathrm{p}<0.009)$. Despite an increased need for salvage therapy, the average cost per month of median survival was $\$ 2412$ per month for SRS alone, $\$ 3220$ per month for SRS+WBRT, and $\$ 4360$ per month for S+SRS ( $\mathrm{p}<0.03$ ). Compared with SRS+WBRT, SRS alone had an average incremental cost savings of $\$ 110$ per patient. Sensitivity analysis confirmed that the average treatment cost of SRS alone remained less than or was comparable to SRS+WBRT over a wide range of costs and treatment efficacies.

Conclusions. Despite an increased need for salvage therapy, patients with newly diagnosed brain metastases treated with SRS alone have similar overall survival and receive more cost-effective care than those treated with SRS+WBRT. Compared with SRS+WBRT, initial management with SRS alone does not result in a higher average cost. (http://thejns.org/doi/abs/10.3171/2014.7.GKS14972)

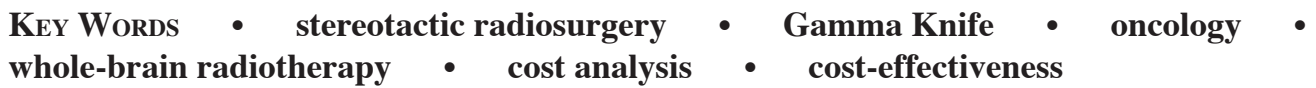

$\mathrm{M}$ ETASTATic brain tumors are the most common intracranial neoplasm, with 170,000 new cases reported per year. ${ }^{16}$ Whole-brain radiotherapy (WBRT) has been a standard treatment option, but ste-

\footnotetext{
Abbreviations used in this paper: ICER $=$ incremental cost-effectiveness ratio; KPS = Karnofsky Performance Scale; MVA= multivariate proportional hazards analysis; $\mathrm{SRS}=$ stereotactic radiosurgery; S+SRS = surgery + SRS; WBRT $=$ whole-brain radiotherapy.
}

reotactic radiosurgery (SRS) is increasingly integrated into the management of this patient population. Three randomized trials comparing SRS alone with SRS and WBRT (SRS+WBRT) showed that adding WBRT to SRS in patients who have up to 4 newly diagnosed brain metastases does not improve overall survival. ${ }^{1,3,5}$ While the addition of WBRT reduces intracranial failure rates, ${ }^{9}$ patients treated with SRS+WBRT are at greater risk of developing learning and memory impairment than patients 
treated with SRS alone. ${ }^{3}$ With more effective systemic therapies and longer survival, reducing the neurocognitive sequelae of treatment is becoming increasingly important.

Whole-brain radiotherapy in addition to SRS reduces intracranial failure rates and the need for salvage therapy, but also causes deleterious effects in patients who may not require additional therapy. The costs of these 2 treatment strategies, with particular attention to the need for subsequent salvage therapies, have not been previously examined. The purpose of this study was to compare the outcomes in patients treated with SRS alone, SRS+WBRT, and surgery followed by SRS (S+SRS) at our institution and to assess the cost-effectiveness of each regimen.

\section{Methods}

\section{Study Design, Participants, and Eligibility Criteria}

Between May 2001 and December 2007, 317 patients received SRS or stereotactic radiotherapy delivered in 5 fractions for newly diagnosed metastatic brain tumors. Patients were divided into 3 treatment subgroups based on initial treatment with the following: 1) SRS alone, 2) SRS+WBRT, and 3) S+SRS. Twenty-one patients with small cell lung cancer, 4 patients with a history of prior malignancy, and 3 patients with incomplete or contradictory records were excluded, leaving 289 evaluable patients. None of the patients had received prior treatment for brain metastases. A retrospective study was performed of patient characteristics, treatment outcomes, and the costs incurred in the treatment of brain metastases, including the cost of primary treatment and all salvage therapies. This study was conducted with the approval of the Peoria Institutional Review Board. A waiver of informed consent was obtained.

All patients had a pathologically confirmed primary malignancy and brain metastases identified on MRI scans. Patient characteristics, including age, sex, Karnofsky Performance Scale (KPS) scores, primary tumor histology, number of brain metastases, primary tumor control, extracranial disease status, and the timing of initial and salvage treatments, were entered into an electronic database. Primary tumor control was defined as definitive surgical resection with negative pathological margins and the absence of imaging findings consistent with systemic disease at the time of surgery. Decisions on treatment with WBRT, surgery, or SRS were made at the discretion of the radiation oncologist and neurosurgeon. Radiosurgery dose prescriptions were based on the Radiation Therapy Oncology Group dose escalation trial ${ }^{13}$ and on our institutional experience. After SRS, patients were followed up with serial clinical examinations and MRI scans every 3 months.

\section{Statistical Analysis}

Statistical analysis was performed using the SPSS software package (version 12.0). Survival curves were plotted using the Kaplan-Meier method and compared using the log-rank test. Overall survival was measured from the date of initial treatment for brain metastases to the date of death from any cause. Time to salvage therapy was measured from the date of SRS to the date of salvage with neurosurgery, SRS, or WBRT. Multivariate proportional hazards analysis (MVA) was used to identify prognostic factors associated with overall survival.

\section{Cost Analysis}

Total treatment costs for each patient were priced based on the 2007 Medicare reimbursement fee schedule as a national standard for therapy performed and assumed from the payer perspective. As a referral center, we could follow up more than $90 \%$ of the patients after initial treatment at our institution or our affiliates. As a result, comprehensive data on the resource use for imaging, hospitalizations, and salvage therapies were available in the medical record.

Treatment costs measured the cost of initial therapy and all salvage treatments for intracranial disease, hospitalization for craniotomies and complications, professional fees, diagnostic imaging, and follow-up care. Expenditures for extracranial disease were not included. The cost of craniotomies performed for radionecrosis were included as incurred. Hospital costs for craniotomies were applied for a median hospital stay of 5 days, on the basis of data extracted from the discharge summaries from the 43 patients who underwent surgery in this study. This figure is comparable to the estimate used by Mehta and colleagues..$^{10}$ Neurosurgical complications were assumed according to the incidence in the medical literature reported by these authors. The cost of WBRT was assumed to be the Medicare reimbursement rate for 35 Gy in 14 fractions, the standard regimen used in our practice. The use of alternative fractionation regimens, such as 30 Gy in 10 fractions or 36 Gy in 18 fractions, can influence costs and this effect was assessed by a sensitivity analysis (see below).

Average cost-effectiveness was defined as the average total treatment cost per month of median survival. ${ }^{15}$ To compare the average cost-effectiveness of each treatment group, the incremental cost-effectiveness ratio (ICER) (shown below), that is, the difference in cost between a trial treatment program and the accepted therapeutic regimen divided by the difference in survival, was calculated as follows:

$$
\begin{aligned}
\operatorname{ICER}= & \left(\text { Cost }_{\text {new therapy }}-\text { Cost }_{\text {current standard }}\right) / \\
& \left(\text { Survival }_{\text {new therapy }}-\text { Survival }_{\text {current standard }}\right)
\end{aligned}
$$

No discount factor was used for the rate of inflation. Because overall survival of patients with metastatic brain tumors is generally $<1$ year, the effect of inflation should be limited.

Differences in average total cost or in average costeffectiveness among the treatment regimens were assessed for statistical significance by t-tests (2-tailed, $\mathrm{p}$ $<0.05)$. Average cost per month of median survival and the average total cost per unadjusted life year were reported to conform to previous studies ${ }^{10,12}$ and to adhere to published guidelines. ${ }^{11,15}$ Outcomes were not adjusted for health-related quality of life to prevent the introduction of potential selection bias. 
A sensitivity analysis was performed to examine the impact of plausible variation in key cost parameters. Reimbursements for SRS, neurosurgery, and WBRT were incrementally increased and decreased by up to $50 \%$ on the basis of the 2007 Medicare fee schedule. Holding all other variables constant, the average total cost and cost-effectiveness of each treatment regimen were calculated in a stepwise manner for each variation in reimbursements.

\section{Patients}

\section{Results}

Patient demographics and their tumor characteristics are shown in Table 1 . The median age of all patients was 64 years. The patients were well balanced in the SRSalone and SRS+WBRT groups in terms of age, sex, KPS score, primary tumor control, and presence of extracranial metastases. Patients treated with SRS alone or S+SRS were somewhat more likely to have fewer metastatic lesions than patients treated with SRS+WBRT.

\section{Outcomes}

The median follow-up length was 9.1 months overall and 27.1 months for patients who were alive at the last

\section{TABLE 1: Patient characteristics*}

\begin{tabular}{|c|c|c|c|}
\hline Variable & SRS Alone & SRS+WBRT & S+SRS \\
\hline median age, yrs (range) & $66(33-91)$ & $59(37-86)$ & $62(30-78)$ \\
\hline total no. of patients & 174 & 90 & 25 \\
\hline \multicolumn{4}{|l|}{ sex } \\
\hline male & $86(49)$ & $43(48)$ & $13(52)$ \\
\hline female & $88(51)$ & $47(52)$ & $12(48)$ \\
\hline \multicolumn{4}{|l|}{ KPS score } \\
\hline $90-100$ & $121(69)$ & $61(68)$ & $15(60)$ \\
\hline$\leq 80$ & $44(25)$ & $23(26)$ & $9(36)$ \\
\hline unknown & $9(5)$ & $6(7)$ & $1(4)$ \\
\hline \multicolumn{4}{|l|}{ primary tumor } \\
\hline NSCLC & $88(51)$ & $58(64)$ & $9(36)$ \\
\hline breast & $27(16)$ & $12(13)$ & $0(0)$ \\
\hline colorectal & $13(8)$ & $4(4)$ & $2(8)$ \\
\hline melanoma & $17(10)$ & $4(4)$ & $9(36)$ \\
\hline renal & $24(14)$ & $6(7)$ & $2(8)$ \\
\hline other & $5(3)$ & $6(7)$ & $3(12)$ \\
\hline \multicolumn{4}{|l|}{ primary tumor status } \\
\hline controlled & $86(49)$ & $38(42)$ & $13(52)$ \\
\hline uncontrolled & $88(51)$ & $52(58)$ & $12(48)$ \\
\hline \multicolumn{4}{|l|}{ extracranial metastases } \\
\hline present & $128(74)$ & $66(73)$ & $15(60)$ \\
\hline absent & $46(26)$ & $24(27)$ & $10(40)$ \\
\hline \multicolumn{4}{|l|}{ no. of brain metastases } \\
\hline 1 & $95(55)$ & $33(37)$ & $15(60)$ \\
\hline $2-3$ & 54 (31) & $28(31)$ & $7(28)$ \\
\hline$>4$ & 25 (14) & $29(32)$ & $3(12)$ \\
\hline
\end{tabular}

\footnotetext{
* Number of patients (\%) are given, unless indicated otherwise. NSCLC
} $=$ non-small cell lung cancer. follow-up. During the first course of therapy, $94.1 \%$ of patients received single-fraction SRS with the Leksell Gamma Knife 4C (Elekta $\mathrm{AB}$ ) and 5.9\% received 5-fraction stereotactic radiotherapy with CyberKnife (Accuray Inc.). For SRS plans, the median dose was 18 Gy (range 12-24 Gy), and all lesions were treated to the $50 \%$ isodose level. Mean coverage was $98 \%$, and the mean selectivity index was $63 \%$.

Complete survival data were available for $96.2 \%$ of the patients. At the time of analysis, 266 patients had died, 12 were alive, and 11 were lost to follow-up. Eight patients died within 1 month of radiosurgery. The Kaplan-Meier plot showed no significant differences in overall survival among the 3 groups ( $\mathrm{p} \sim 0.2)$ (Fig. 1). Median overall survival was 9.8 months (95\% CI 7.2-11.8) for SRS alone, $7.4(95 \%$ CI 5.6-10.3) for SRS+WBRT, and $10.6(95 \%$ CI 6.7-17.0) for S+SRS. One-year overall survival was $41.6 \%$ (95\% CI $33.6 \%-48.9 \%$ ) for SRS alone, $31.5 \%(95 \%$ CI $22.8 \%-41.1 \%$ ) for SRS+WBRT, and $43.3 \%$ (95\% CI $23.7 \%-63.0 \%$ ) for S+SRS.

Multivariate proportional hazards analysis showed that female sex, KPS score, primary tumor control, absence of extracranial metastases, and number of brain metastases were all statistically significant predictors of overall survival (Table 2). Age and primary tumor histology were not statistically significant. Although patients receiving SRS+WBRT were somewhat more likely to have a greater number of brain metastases than patients treated with SRS alone, the patient subgroups were otherwise reasonably matched with regard to significant prognostic variables.

After the first treatment course, salvage therapy was administered to $43 \%$ of patients treated with SRS alone, whereas $26 \%$ of patients were treated with SRS+WBRT $(p<0.009)$. Salvage therapies included repeat SRS, crani-

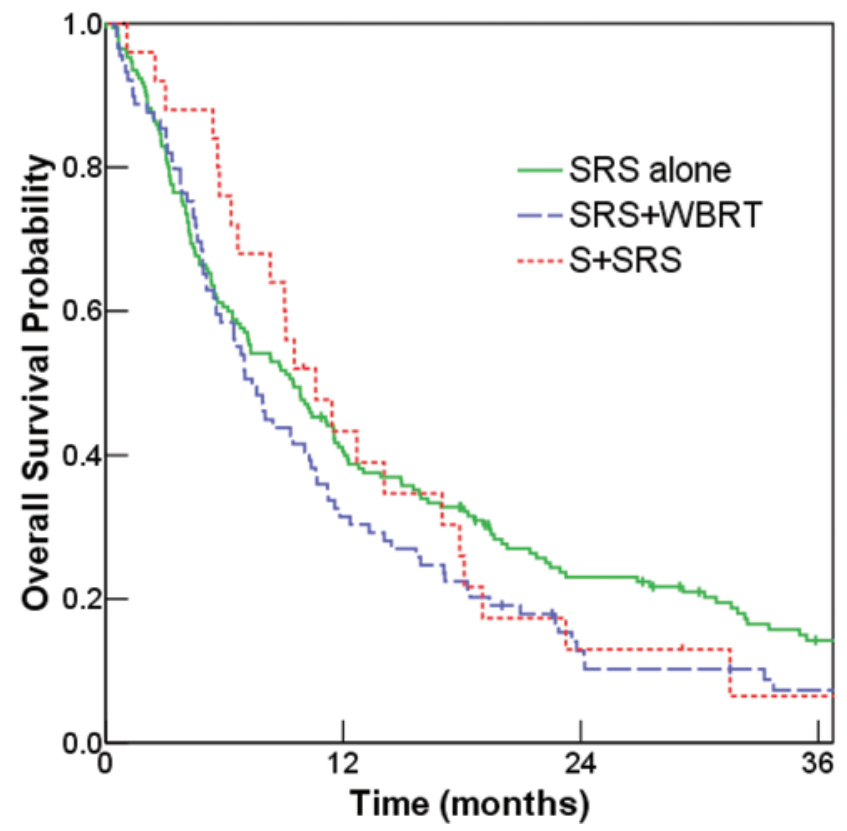

Fig. 1. Overall survival by treatment group. The Kaplan-Meier curves showed no statistically significant association between the initial course of treatment administered and overall survival $(p \sim 0.2)$. 
TABLE 2: Multivariate proportional hazards analysis*

\begin{tabular}{lcc}
\hline \multicolumn{1}{c}{ Variable } & Hazard Ratio $(95 \% \mathrm{Cl})$ & p Value \\
\hline female sex & $0.68(0.51-0.90)$ & 0.007 \\
KPS score & 1.00 & $\mathrm{NA}$ \\
$\quad 90-100$ & $1.40(1.13-1.71)$ & 0.004 \\
$\quad \mathbf{8 0}$ & $1.11(1.03-1.19)$ & 0.006 \\
no. of brain metastases & $0.50(0.35-0.71)$ & $<0.0001$ \\
primary tumor controlled & $1.39(1.01-1.91)$ & 0.043 \\
$\begin{array}{l}\text { absence of extracranial } \\
\text { metastases }\end{array}$ & & \\
\hline
\end{tabular}

* NA = not applicable.

otomy, and WBRT. The mean time to first salvage therapy was 10.6 months in the SRS-alone group and 9.4 months in the SRS+WBRT group. For patients who showed intracranial disease progression after SRS alone, $28 \%$ were treated with WBRT during follow-up. As a result, only $12 \%$ of patients in the SRS-alone group overall received delayed WBRT.

\section{Cost Analysis}

Figure 2 depicts the average total cost of treatment for each group. All costs reflect the cost of primary therapy for brain metastases, hospital charges for neurosurgery, professional fees, imaging, and all ensuing salvage therapies, as incurred. The average total cost for patients treated with SRS alone was $\$ 23,712$ (median $\$ 17,692$ ) versus $\$ 23,822$ for SRS+WBRT (median $\$ 18,825$ ). The average total cost for $\mathrm{S}+\mathrm{SRS}$ was $\$ 46,299$ (median $\$ 45,179$ ). When the cost of salvage therapies was included, the average total costs were not statistically significantly different between SRS alone and SRS+WBRT ( $\mathrm{p} \sim 0.9)$.

Average cost-effectiveness, defined as the average total cost per month median survival, was $\$ 2412$ per month for SRS alone, $\$ 3220$ per month for SRS+WBRT, and $\$ 4360$ per month for S+SRS, which were all statistically significantly different ( $\mathrm{p}<0.03$ for all comparisons). Cost-effectiveness measures were also compared using an equivalent median survival in all 3 groups of 9.8 months, which was the median survival of patients treated with SRS alone. Assuming this median survival time, the normalized average cost-effectiveness was $\$ 2423$ per month for SRS+WBRT and $\$ 4710$ for S+SRS, whereas that of SRS alone was $\$ 2412$ per month.

In this study, compared with SRS+WBRT, patients treated with SRS alone had a 2.4-month longer median survival and an incremental savings of $\$ 110$ per patient. An ICER could not be calculated for SRS alone, given that treatment with SRS alone was both less costly and resulted in longer median survival than SRS+WBRT treatment. Patients treated with S+SRS had an ICER of $\$ 6976$ per extra month survival, reflecting an average incremental cost of $\$ 22,477$ and 3.2 months longer median survival than those treated with SRS+WBRT. The average total cost per unadjusted life year was $\$ 28,946$ for SRS alone, $\$ 38,645$ for SRS+WBRT, and $\$ 52,320$ for S+SRS.

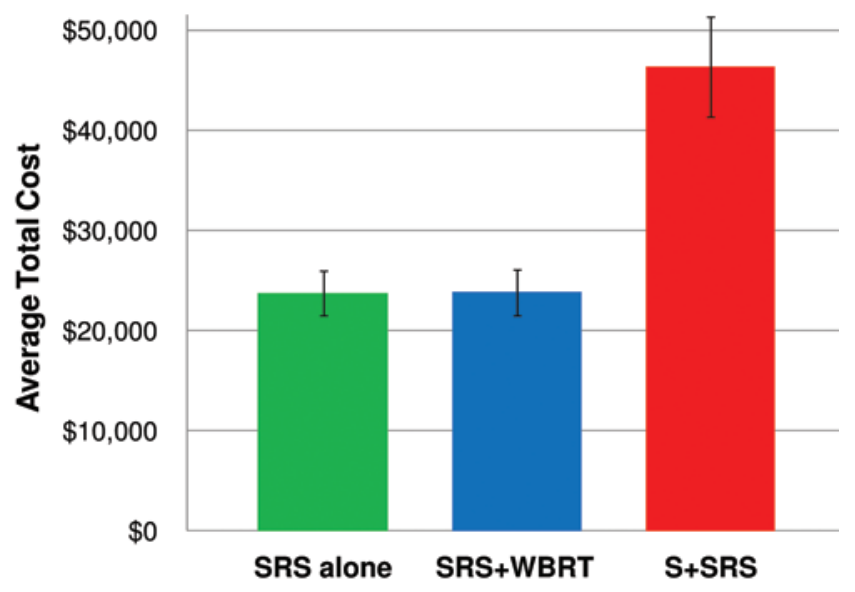

FIG. 2. Average total treatment costs of SRS alone, SRS+WBRT, and S+SRS. The error bars denote the $95 \%$ confidence intervals.

\section{Sensitivity Analysis}

To evaluate the impact of discrete variations in the reimbursement rates for SRS, craniotomy, and WBRT on the average total cost and cost-effectiveness of each treatment regimen, a sensitivity analysis was performed. For changes in the reimbursement rate for SRS and craniotomy by up to about $50 \%$, SRS alone remained more costeffective than SRS+WBRT or S+SRS. Table 3 shows the effects of changes in reimbursement rates for WBRT and of using alternative fractionation schedules on treatment costs. Again, SRS alone remained more cost-effective than SRS+WBRT for all of the examined WBRT reimbursement rates. For all comparisons, the average total cost for SRS alone was either less expensive than or not significantly different from SRS+WBRT.

\section{Discussion}

Whole-brain radiotherapy remains a standard treatment for metastatic brain tumors despite its protracted duration and side effects, including radiation-induced dementia. In contrast, SRS can be performed in a single day, can provide more rapid symptom palliation, and can minimize delays in systemic chemotherapy. In addition, patients treated with SRS alone have significantly fewer problems with memory, concentration, fatigue, and mood disorders than patients treated with SRS alone. ${ }^{3,6}$ By withholding upfront WBRT in appropriately selected patients, clinicians may delay or completely avoid the neurocognitive toxicity of this treatment.

Treatment with SRS alone results in higher intracranial failure rates and in an increased need for salvage therapy than SRS+WBRT treatment. ${ }^{14,17}$ The addition of WBRT, however, has not been shown to improve overall survival in multiple series and 3 randomized trials. ${ }^{1,3,5,18}$ In a retrospective series of 105 patients, Sneed et al. reported that $40 \%$ of patients managed with SRS alone had received salvage therapy including WBRT, partial brain radiotherapy, repeat SRS, or resection, compared with $19 \%$ of patients treated with SRS+WBRT. ${ }^{17}$ Salvage WBRT was needed in $26 \%$ of the patients treated with 
M. D. Hall et al.

TABLE 3: Sensitivity analysis*

\begin{tabular}{|c|c|c|c|c|c|c|c|c|c|}
\hline \multirow[b]{2}{*}{ Variable } & \multicolumn{9}{|c|}{ Dose Regimen/Reimbursement Rate } \\
\hline & $-50 \%$ & $-25 \%$ & $-10 \%$ & $35 \mathrm{~Gy} / 14 \mathrm{Fx}$ & $+10 \%$ & $+25 \%$ & $+50 \%$ & $30 \mathrm{~Gy} / 10 \mathrm{Fx}$ & $36 \mathrm{~Gy} / 18 \mathrm{Fx}$ \\
\hline WBRT cost & 2219 & 3329 & 3994 & 4438 & 4882 & 5548 & 6657 & 3467 & 5138 \\
\hline SRS alone & 23,419 & 23,565 & 23,653 & 23,712 & 23,771 & 23,859 & 24,005 & 23,584 & 23,804 \\
\hline SRS+WBRT & 21,504 & 22,663 & 23,358 & 23,822 & 24,285 & 24,981 & 26,139 & 22,808 & 24,553 \\
\hline S+SRS & 45,855 & 46,077 & 46,210 & 46,299 & 46,387 & 46,521 & 46,742 & 46,104 & 46,439 \\
\hline SRS alone & 2382 & 2397 & 2406 & 2412 & 2418 & 2427 & 2442 & 2399 & 2422 \\
\hline SRS+WBRT & 2907 & 3064 & 3158 & 3220 & 3283 & 3377 & 3534 & 3083 & 3319 \\
\hline S+SRS & 4318 & 4339 & 4352 & 4360 & 4368 & 4381 & 4402 & 4342 & 4373 \\
\hline
\end{tabular}

* The average total cost and the average cost-effectiveness (both in US\$) per month median survival of SRS alone, SRS+WBRT, and S+SRS were calculated for each stepwise variation in the reimbursement rate for WBRT. The shaded column indicates the cost figures used in our analysis, which assumed that WBRT was given in 35 Gy/14 fractions. The adjusted average total cost and average cost-effectiveness are reported for each specified level of reimbursement. $\mathrm{Fx}=$ fractions.

SRS alone. In our series, $43 \%$ of patients treated with SRS alone and $26 \%$ treated with SRS+WBRT required salvage therapy. For patients who developed intracranial disease progression after SRS alone, only $28 \%$ received delayed WBRT. As a result, $88 \%$ of patients managed with SRS alone were spared from WBRT and its sequelae. This finding was shaped by the clinical preferences of our treatment team and cannot be easily compared with other reports.

The current American Society for Radiation Oncology evidence-based guidelines support SRS alone for patients with 1-4 newly diagnosed metastatic brain tumors. ${ }^{19}$ In a prospective trial of 1194 patients, Yamamoto et al. reported that patients with 5-10 brain metastases had overall survival comparable to that in patients with 2-4 lesions when treated with SRS alone, suggesting that SRS alone may be suitable for patients having up to 10 lesions. ${ }^{20}$ In the absence of an overall survival advantage, risk of distant failure, neurological deterioration, and death due to intracranial progression are the rationale for the use of WBRT in this setting. When salvage therapy can be readily administered, however, increasing evidence indicates that WBRT may not be needed in all cases. Stereotactic radiosurgery alone may be justified in patients with good KPS scores and small intracranial tumor volume.

Cost, however, is another increasingly important consideration. Mehta et al. found that SRS+WBRT was a cost-effective treatment regimen in patients with a single brain metastasis, with an average cost per week of survival of \$270 for SRS+WBRT compared with \$310 for WBRT alone. ${ }^{10}$ To our knowledge, ours is the first study to directly analyze the average total costs and cost-effectiveness of SRS alone and SRS+WBRT in patients with 1 or more brain metastases using patient-level data and actual costing, although modeling has been previously used. ${ }^{7}$

Treatment with SRS alone or with SRS+WBRT of patients with 1 or more newly diagnosed brain metastases resulted in similar overall survival. Despite an increased need for salvage therapy, our analysis demonstrated that patients treated with SRS alone received more cost-effective therapy than those treated with SRS+WBRT. In addition, $57 \%$ of patients treated with SRS alone did not require any salvage therapy, and in $88 \%$ of these patients, WBRT was avoided altogether. The addition of WBRT did not improve overall survival in any patient subgroup and did not reduce average total treatment cost when compared with the average cost of SRS alone.

Although no statistically significant difference between the average total cost for SRS alone and SRS+WBRT was observed in our series, the sensitivity analysis demonstrated that variations in reimbursement rates can influence costs. Two findings in particular deserve mention. First, treatment with S+SRS entailed significantly greater average total costs than SRS alone and SRS+WBRT. However, patients treated with neurosurgery often present with larger, more symptomatic lesions, where rapid palliation is needed. Although invasive and more costly, neurosurgery is clearly indicated in selected patients and should not be restricted where SRS and WBRT are not comparable alternatives.

Second, the WBRT regimen administered significantly impacted the cost difference between SRS alone and SRS+WBRT. The sensitivity analysis showed that if WBRT were given at a dose of 35 Gy in 14 fractions, the average total cost would be $\$ 23,822$ per patient for SRS+WBRT versus $\$ 23,712$ for SRS alone. In our analysis, WBRT costs did not include charges for 3D conformal treatment planning and dose-volume histogram analysis, which were not routinely performed for WBRT during the study period. According to the 2014 Medicare fee schedule, reimbursement for this regimen has increased by more than $\$ 2500$ per course, caused in part by the use of such treatment planning techniques. Changes in reimbursement rates since 2007 have resulted in rising fees for WBRT and lower reimbursements for single-fraction SRS than were used in our analysis, which can dramatically impact the average total treatment cost. Newer strategies to preserve neurocognitive function, including meman- 
tine $^{2}$ and hippocampal-sparing intensity-modulated radiation therapy, ${ }^{4}$ also raise the treatment costs for WBRT.

Treatment decisions for patients with metastatic brain tumors should be made on an individualized basis to maximize positive clinical outcomes and patient quality of life and should not be determined solely by cost. Our analysis showed that patients treated with SRS alone received more cost-effective therapy than those treated with SRS+WBRT. Only prospective clinical trials can provide conclusive evidence for which patients can be safely managed with SRS alone. However, our results demonstrated that excess cost is not a valid reason to select SRS+WBRT instead of SRS alone.

This study is a retrospective analysis of patients treated at a single institution and is subject to limitations. First, selection bias is inevitable in retrospective series and may have influenced the treatments received. Although worthy of comment, incurred costs can still be accurately collected and reported with meticulous follow-up, as is shown here. Stereotactic radiosurgery alone is known to better preserve neurocognition and to result in better quality of life than treatment with SRS+WBRT.3,8 As a result, given their comparable average costs, SRS alone should logically be noninferior to SRS+WBRT in terms of cost per life year and cost per quality-adjusted life year.

Second, the validity of the cost analysis is directly dependent on the assumptions used; deviations in Medicare reimbursement rates and the selection of salvage treatments, in particular, may alter the results. For this study, the Medicare fee schedule was selected to minimize heterogeneity in reimbursement rates for the same services rendered. As a result, the reported cost estimates in this study are not influenced by differences in institutional billing practices or patient insurance plans. On the other hand, the Medicare basis for cost estimates would not perfectly recapitulate the fees paid at other facilities and times.

Despite these limitations, large patient numbers, balanced demographics, and the use of actual treatment costs rather than modeling should limit potential bias. In addition, the results presented here are based on mature and comprehensive follow-up data regarding overall survival and salvage therapy administered, which may have helped ensure that treatment outcomes and costs were accurate. Based on the mounting evidence that SRS alone results in overall survival comparable to other treatments and better preserves neurocognition, this approach has been increasingly used at our institution as a first-line therapy for patients who have newly diagnosed brain metastases and good KPS scores.

\section{Conclusions}

Our findings suggest that patients treated with SRS alone have similar overall survival and receive more costeffective therapy than patients treated with SRS+WBRT. Treatment with SRS alone resulted in average treatment costs comparable to SRS+WBRT despite an increased need for salvage therapy. Clinical trials must be performed to validate the use of SRS alone in patients with brain metastases.

\section{Disclosure}

The authors report no conflict of interest concerning the materials or methods used in this study or the findings specified in this paper.

Author contributions to the study and manuscript preparation include the following. Conception and design: MD Hall, JL McGee. Acquisition of data: MD Hall, JL McGee, KA Hall. Analysis and interpretation of data: all authors. Drafting the article: all authors. Critically revising the article: all authors. Reviewed submitted version of manuscript: all authors. Approved the final version of the manuscript on behalf of all authors: MD Hall. Statistical analysis: MD Hall. Administrative/technical/material support: JL McGee. Study supervision: MD Hall, JL McGee.

\section{References}

1. Aoyama H, Shirato H, Tago M, Nakagawa K, Toyoda T, Hatano $\mathrm{K}$, et al: Stereotactic radiosurgery plus whole-brain radiation therapy vs stereotactic radiosurgery alone for treatment of brain metastases: a randomized controlled trial. JAMA 295:2483-2491, 2006

2. Brown PD, Pugh S, Laack NN, Wefel JS, Khuntia D, Meyers $\mathrm{C}$, et al: Memantine for the prevention of cognitive dysfunction in patients receiving whole-brain radiotherapy: a randomized, double-blind, placebo-controlled trial. Neuro Oncol 15:1429-1437, 2013

3. Chang EL, Wefel JS, Hess KR, Allen PK, Lang FF, Kornguth DG, et al: Neurocognition in patients with brain metastases treated with radiosurgery or radiosurgery plus whole-brain irradiation: a randomised controlled trial. Lancet Oncol 10:1037-1044, 2009

4. Gondi V, Tomé WA, Mehta MP: Why avoid the hippocampus? A comprehensive review. Radiother Oncol 97:370-376, 2010

5. Kocher M, Soffietti R, Abacioglu U, Villà S, Fauchon F, Baumert BG, et al: Adjuvant whole-brain radiotherapy versus observation after radiosurgery or surgical resection of one to three cerebral metastases: results of the EORTC 22952-26001 study. J Clin Oncol 29:134-141, 2011

6. Kondziolka D, Niranjan A, Flickinger JC, Lunsford LD: Radiosurgery with or without whole-brain radiotherapy for brain metastases: the patients' perspective regarding complications. Am J Clin Oncol 28:173-179, 2005

7. Lal LS, Byfield SD, Chang EL, Franzini L, Miller LA, Arbuckle R, et al: Cost-effectiveness analysis of a randomized study comparing radiosurgery with radiosurgery and whole brain radiation therapy in patients with 1 to 3 brain metastases. Am J Clin Oncol 35:45-50, 2012

8. Lester-Coll NH, Dosoretz AP, Yu JB: Decision analysis of stereotactic radiation surgery versus stereotactic radiation surgery and whole-brain radiation therapy for 1 to 3 brain metastases. Int J Radiat Oncol Biol Phys 89:563-568, 2014

9. Lo SS, Chang EL, Suh JH: Stereotactic radiosurgery with and without whole-brain radiotherapy for newly diagnosed brain metastases. Expert Rev Neurother 5:487-495, 2005

10. Mehta M, Noyes W, Craig B, Lamond J, Auchter R, French M, et al: A cost-effectiveness and cost-utility analysis of radiosurgery vs. resection for single-brain metastases. Int J Radiat Oncol Biol Phys 39:445-454, 1997

11. Russell LB, Gold MR, Siegel JE, Daniels N, Weinstein MC: The role of cost-effectiveness analysis in health and medicine. JAMA 276:1172-1177, 1996

12. Rutigliano MJ, Lunsford LD, Kondziolka D, Strauss MJ, Khanna V, Green M: The cost effectiveness of stereotactic radiosurgery versus surgical resection in the treatment of solitary metastatic brain tumors. Neurosurgery 37:445-455, 1995

13. Shaw E, Scott C, Souhami L, Dinapoli R, Kline R, Loeffler J, et al: Single dose radiosurgical treatment of recurrent previ- 
ously irradiated primary brain tumors and brain metastases: final report of RTOG protocol 90-05. Int J Radiat Oncol Biol Phys 47:291-298, 2000

14. Shehata MK, Young B, Reid B, Patchell RA, St. Clair W, Sims J, et al: Stereotactic radiosurgery of 468 brain metastases $\leq 2$ $\mathrm{cm}$ : implications for SRS dose and whole brain radiation therapy. Int J Radiat Oncol Biol Phys 59:87-93, 2004

15. Siegel JE, Weinstein MC, Russell LB, Gold MR: Recommendations for reporting cost-effectiveness analyses. JAMA 276:1339-1341, 1996

16. Smith ML, Lee JY: Stereotactic radiosurgery in the management of brain metastasis. Neurosurg Focus 22(3):E5, 2007

17. Sneed PK, Lamborn KR, Forstner JM, McDermott MW, Chang S, Park E, et al: Radiosurgery for brain metastases: is whole brain radiotherapy necessary? Int J Radiat Oncol Biol Phys 43:549-558, 1999

18. Sneed PK, Suh JH, Goetsch SJ, Sanghavi SN, Chappell R, Buatti JM, et al: A multi-institutional review of radiosurgery alone vs. radiosurgery with whole brain radiotherapy as the initial management of brain metastases. Int J Radiat Oncol Biol Phys 53:519-526, 2002

19. Tsao MN, Rades D, Wirth A, Lo SS, Danielson BL, Gaspar
LE, et al: Radiotherapeutic and surgical management for newly diagnosed brain metastasis(es): an American Society for Radiation Oncology evidence-based guideline. Pract Radiat Oncol 2:210-225, 2012

20. Yamamoto M, Serizawa T, Shuto T, Akabane A, Higuchi Y, Kawagishi J, et al: Stereotactic radiosurgery for patients with multiple brain metastases (JLGK0901): a multi-institutional prospective observational study. Lancet Oncol 15:387-395, 2014

Manuscript submitted May 16, 2014.

Accepted July 25, 2014

Abstract previously presented at the 17th International Leksell Gamma Knife Society Meeting in New York, NY, on May 13, 2014.

Please include this information when citing this paper: DOI: 10.3171/2014.7.GKS14972.

Address correspondence to: Matthew D. Hall, M.D., M.B.A., Department of Radiation Oncology, City of Hope National Medical Center, 1500 E. Duarte Rd., Duarte, CA 91010. email: mhall@coh. org. 